\title{
Temporal and spatial changes of monocarboxylate transporter 4 expression in the hippocampal CA1 region following transient forebrain ischemia in the Mongolian gerbil
}

\author{
DAE YOUNG YOO ${ }^{*}$, JOON HA PARK ${ }^{2 *}$, KWON YOUNG LEE ${ }^{3}$, HYUN JUNG KWON $^{4}$, \\ HYO YOUNG JUNG ${ }^{1}$, JONG WHI KIM ${ }^{1}$, DAE WON KIM ${ }^{4}$, JUNG HOON $\mathrm{CHOI}^{3}$, \\ SEUNG MYUNG MOON ${ }^{5}$, YEO SUNG YOON ${ }^{1}$, MOO-HO WON ${ }^{2}$ and IN KOO HWANG ${ }^{1}$
}

\begin{abstract}
${ }^{1}$ Department of Anatomy and Cell Biology, College of Veterinary Medicine and Research Institute for Veterinary Science, Seoul National University, Seoul 08826; ${ }^{2}$ Department of Neurobiology, School of Medicine;

${ }^{3}$ Department of Anatomy, College of Veterinary Medicine, Kangwon National University, Chuncheon, Gangwon 24341;

${ }^{4}$ Department of Biochemistry and Molecular Biology, Research Institute of Oral Sciences, College of Dentistry, Gangneung-Wonju National University, Gangneung, Gangwon 25457; ${ }^{5}$ Department of Neurosurgery, Dongtan Sacred

Heart Hospital, College of Medicine, Hallym University, Hwaseong, Gyeonggi 18450, Republic of Korea
\end{abstract}

Received March 3, 2016; Accepted February 13, 2017

DOI: $10.3892 / \mathrm{mmr} .2017 .6508$

\begin{abstract}
Transient forebrain ischemia depletes glucose and oxygen levels in the brain. In this pathological condition, lactate serves an important role in cellular metabolism as the end product of glycolysis. The present study investigated the expression of monocarboxylate transporter 4 (MCT4) in lactate metabolism in the hippocampal CA1 region following induction of transient forebrain ischemia. MCT4 immunoreactivity was detected in CA1 pyramidal cells of the sham-operated group. Animals from the ischemic group exhibited a transient decrease in MCT4 immunoreactivity in the CA1 region between $30 \mathrm{~min}$ and $3 \mathrm{~h}$ following ischemia compared with the sham-operated group. The initial decrease in immunoreactivity observed between $30 \mathrm{~min}$ and $3 \mathrm{~h}$ following ischemia was followed by an increase at 2 days after the treatment. A significant increase in MCT4 immunoreactivity levels was observed 2 days after ischemia compared with the sham-operated group. Limited MCT4 immunoreactivity was observed in the pyramidal neurons 3 days
\end{abstract}

Correspondence to: Professor Moo-Ho Won, Department of Neurobiology, School of Medicine, Kangwon National University, 1 Kangwondaehak-gil, Chuncheon, Gangwon 24341, Republic of Korea E-mail: mhwon@kangwon.ac.kr

Professor In Koo Hwang, Department of Anatomy and Cell Biology, College of Veterinary Medicine, Seoul National University, 1 Gwanak-ro, Seoul 08826, Republic of Korea

E-mail: vetmed2@snu.ac.kr

*Contributed equally

Key words: transient forebrain ischemia, monocarboxylate transporter 4, pyramidal cell, astrocytes, gerbils after ischemia. At 4-10 days after ischemia, MCT4 immunoreactivity was detected in the strata radiatum, oriens and pyramidale. Furthermore, MCT4 immunoreactivity levels in the CA1 region exhibited a time-dependent increase following ischemia. The results indicated that there were transient alterations observed in the localization of MCT4 following the induction of ischemia, and further studies are required to investigate the association between MCT4 expression and lactate metabolism in providing energy to the post-ischemic brain.

\section{Introduction}

The brain uses glucose as a primary energy substrate under physiological conditions, however, the brain is also capable of oxidizing other intermediate metabolites, such as lactate $(1,2)$. The reduced glucose and oxygen levels induced by ischemia causes a rapid reduction in ATP supplies by increasing the rate of anaerobic glycolysis, which leads to lactate accumulation, rather than initiating the respiratory chain reaction by the tricarboxylic acid cycle $(3,4)$. In the brain, lactate is catalyzed by several monocarboxylate transporters (MCTs), which rapidly transport lactate across the plasma membrane (5). Among MCTs, MCT1, MCT2 and MCT4 isoforms have been previously detected in the brain (6). MCT1 has been predominantly observed in the endothelial cells of microvessels and also in glial cells (7-9). In contrast, MCT2 and MCT4 isoforms have been observed in neurons and astrocytes (9-14). Several studies have reported cell-specific changes in the expression of MCTs occurring in different rat models of ischemia (14-17). In a previous study of the authors, MCT4 was indicated to be expressed in the pyramidal cells of the gerbil hippocampus following the induction of transient forebrain ischemia. In addition, the authors indicated that MCT4 may be one of the factors that serves a key role in ischemic preconditioning (13). However, there are few other studies investigating the changes 
of hippocampal MCTs isoforms following the induction of transient forebrain ischemia. To improve the understanding of the role of MCT4 in transient forebrain ischemia, the present study investigated the temporal and spatial changes of MCT4 in the hippocampal CA1 region of the Mongolian gerbil at several time points following ischemia.

\section{Materials and methods}

Experimental animals. A total of 85 male Mongolian gerbils (3-months-old; 50-60 g) were purchased from Japan SLC, Inc. (Shizuoka, Japan). They were housed under standard conditions with adequate temperature $\left(22^{\circ} \mathrm{C}\right)$ and humidity $(60 \%)$ control, a $12 \mathrm{~h}$ light $/ 12 \mathrm{~h}$ dark cycle and had free access to food and water. The handling and care of the animals conformed to the guidelines established to comply with current international laws and policies [NIH Guide for the Care and Use of Laboratory Animals, NIH Publication No. 85-23, 1985, revised 1996 (18)], and were approved by the Institutional Animal Care and Use Committee of Seoul National University (Seoul, Korea). All of the experiments were conducted with an effort to minimize the number of animals used and the suffering caused by the procedures employed in the present study.

Induction of transient forebrain ischemia. The animals were anesthetized with a mixture of $2.5 \%$ isoflurane (Hana Pharmaceutical Co., Ltd., Seoul, South Korea) in 33\% oxygen and $67 \%$ nitrous oxide. Bilateral common carotid arteries were isolated and occluded using non-traumatic aneurysm clips. The complete interruption of blood flow was confirmed by observing the central artery in retinae using an ophthalmoscope (HEINE K180 ${ }^{\circledR}$; Heine Optotechnik, Herrsching, Germany). Following 5 min of occlusion, the aneurysm clips were removed from the common carotid arteries. Blockade of blood flow by occlusion of the common carotid arteries was not performed in the animals of the sham-operated group. The body (rectal) temperature under free-regulating or normothermic $\left(37 \pm 0.5^{\circ} \mathrm{C}\right)$ conditions was monitored with a rectal temperature probe (TR-100; Fine Science Tools GmbH, Foster City, CA, USA) and maintained using a thermometric blanket prior to, during and following surgery until the animals completely recovered from anesthesia. Thereafter, animals were kept on the thermal incubator to maintain the body temperature of animals until the animals were euthanized.

Immunohistochemistry. For histology, sham- and ischemia-operated animals ( $\mathrm{n}=5$ per group) were anesthetized with $1 \mathrm{~g} / \mathrm{kg}$ urethane (Sigma-Aldrich; Merck KGaA, Darmstadt, Germany) at various time points following ischemia/reperfusion and perfused transcardially with $0.1 \mathrm{M}$ PBS ( $\mathrm{pH} 7.4$ ), which was followed by $4 \%$ paraformaldehyde in $0.1 \mathrm{M}$ phosphate-buffered saline ( $\mathrm{pH} 7.4)$. The brains were removed and post-fixed in $4 \%$ paraformaldehyde for $12 \mathrm{~h}$ at $25^{\circ} \mathrm{C}$. The brain tissues were cryoprotected by infiltration with $30 \%$ sucrose overnight at $4^{\circ} \mathrm{C}$. Brain sections $(30 \mu \mathrm{m})$ in coronal plane were serially cut using a cryostat (Leica Microsystems $\mathrm{GmbH}$, Wetzlar, Germany). The sections were collected into 6-well plates containing PBS for further processes.

To ensure that the immunohistochemical data were comparable between groups, the sections were carefully processed under the same conditions. The tissue sections were selected between $1.4 \mathrm{~mm}$ and $2.0 \mathrm{~mm}$ posterior to the bregma in reference to a gerbil brain atlas (19) for each animal. A total of 5 sections per group, $90 \mu \mathrm{m}$ apart from each other, were sequentially treated with $0.3 \%$ hydrogen peroxide in PBS for $30 \mathrm{~min}$ and $10 \%$ normal goat serum (Vector Laboratories, Inc., Burlingame, CA, USA) in $0.05 \mathrm{M} \mathrm{PBS}$ for $30 \mathrm{~min}$ at $25^{\circ} \mathrm{C}$. They were subsequently incubated with rabbit anti-MCT4 antibodies (1:200; catalog no. sc-50329; Santa Cruz Biotechnology, Inc., Dallas, TX, USA) overnight at $25^{\circ} \mathrm{C}$, followed by treatment with biotinylated goat anti-rabbit $\mathrm{IgG}$ for $2 \mathrm{~h}$ at $25^{\circ} \mathrm{C}(1: 200$; catalog no. BA-1000; Vector Laboratories, Inc.) and a streptavidin-peroxidase complex (catalog no. PK-6100; Vector Laboratories, Inc.). Sections were subsequently visualized by reaction with 3,3'-diaminobenzidine tetrachloride (Sigma-Aldrich; Merck KGaA) in 0.1 M Tris- $\mathrm{HCl}$ buffer ( $\mathrm{pH}$ 7.2) and mounted on gelatin-coated slides. Sections were mounted in Canada Balsam (Kanto Chemical Co., Inc., Tokyo, Japan) following dehydration.

Analysis of the regions of interest in the stratum oriens, stratum pyramidale and stratum radiatum of the hippocampal CA1 region was performed using an image analysis system and ImageJ software version 1.50 (National Institutes of Health, Bethesda, MD, USA). Images were calibrated into an array of $512 \times 512$ pixels corresponding to a tissue area of 140x140 $\mu \mathrm{m}$ (x40 magnification). Each pixel resolution was 256 gray levels. The intensity of MCT4 immunoreactivity was evaluated by relative optical density (ROD), which was obtained following transformation of the mean gray level using the following formula: ROD=log (256/mean gray level). ROD of background was determined in unlabeled portions and this value was subtracted for correction, yielding high ROD values in the presence of preserved structures and low values following structural loss using Image J software version 1.50 (National Institutes of Health). A ratio of the ROD was calibrated as percentage compared with the control.

Double immunofluorescence. To confirm the co-localization of MCT4 and glial fibrillary acidic protein (GFAP) in the brain, sections taken 7 days following ischemia were processed under the same conditions as the immunohistochemistry analysis prior to addition of the primary antibody. Double immunofluorescence staining for rabbit anti-MCT4 (1:50) and mouse anti-GFAP (1:500; catalog no. AB5804; EMD Millipore, Billerica, MA, USA) was performed. The sections were incubated in the mixture of antisera overnight at $25^{\circ} \mathrm{C}$. Following three washes for 10 min each with PBS, they were subsequently incubated in a mixture of $\mathrm{Cy} 3$-conjugated donkey anti-rabbit IgG (1:600; catalog no. 711-165-152; Jackson ImmunoResearch Laboratories, Inc., West Grove, PA, USA) and FITC-conjugated donkey anti-mouse IgG (1:600; catalog no. 715-095-151; Jackson ImmunoResearch Laboratories, Inc.) for $2 \mathrm{~h}$ at $25^{\circ} \mathrm{C}$. The immunoreactions were observed under a confocal microscope (LSM 510 META NLO; Zeiss GmbH, Jena, Germany).

Western blot analysis. To confirm the changes in the expression of MCT4 in the hippocampus, animals in sham- and ischemia-operated animals $(n=5)$ were sacrificed and western blot analysis was performed. Following sacrifice and removal of tissues, the tissues were cut into $500 \mu \mathrm{m}$ thick sections on a 

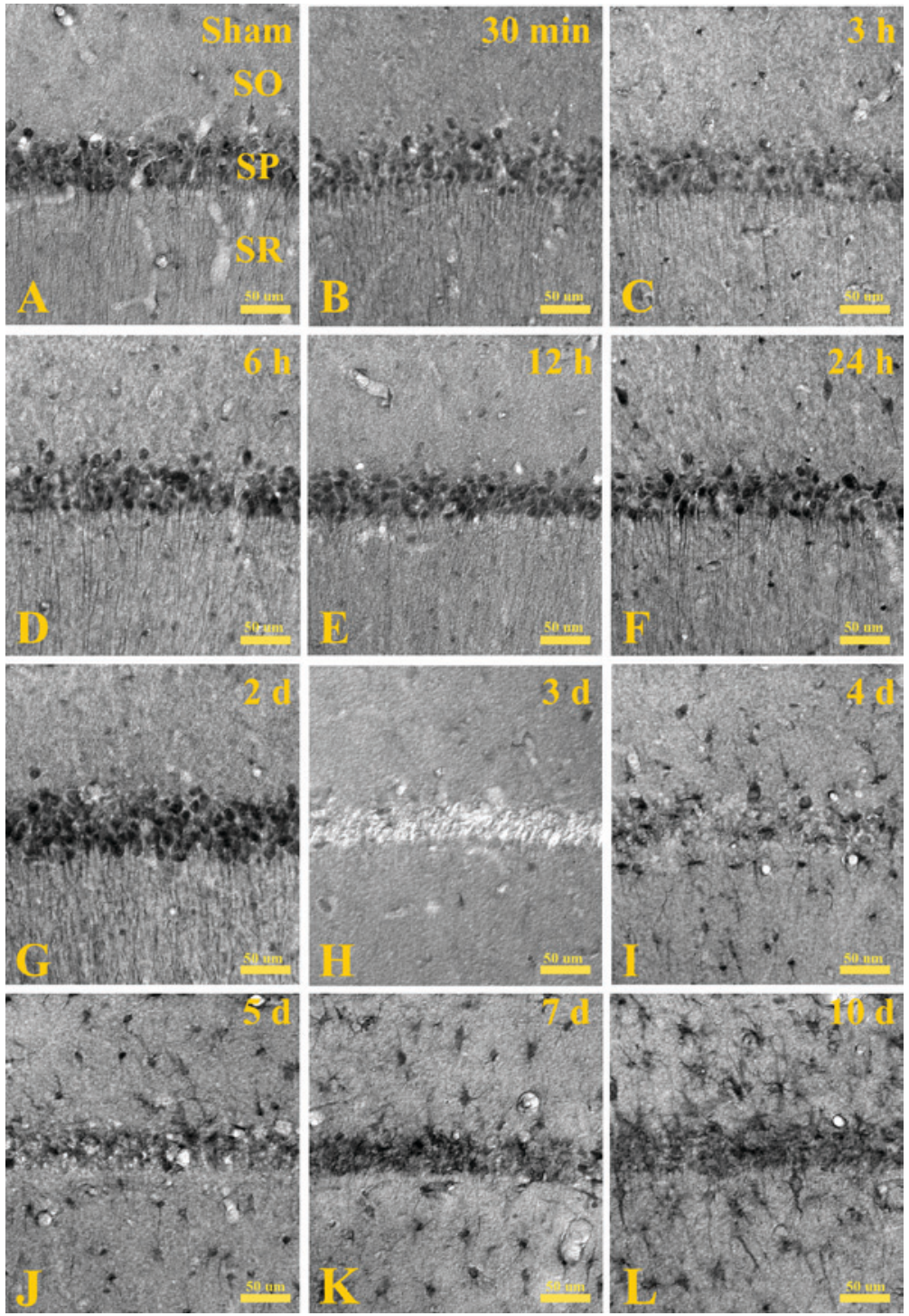

Figure 1. Immunohistochemical staining for MCT4 in the hippocampal CA1 region of sham-operated and operated groups. (A) Sham-operated group. MCT4 staining in ischemia-operated group at (B) $30 \mathrm{~min},(\mathrm{C}) 3 \mathrm{~h},(\mathrm{D}) 6 \mathrm{~h},(\mathrm{E}) 12 \mathrm{~h},(\mathrm{~F}) 24 \mathrm{~h},(\mathrm{G}) 2$ days, (H) 3 days, (I) 4 days, (J) 5 days, (K) 7 days and (L) 10 days following ischemia. MCT4 immunoreactivity is observed in the SP of the sham-operated group. MCT4 exhibits strong immunoreactivity in the SP 2 days following ischemia. However, MCT4 immunoreactivity is decreased in the same region 3 days following ischemia. Thereafter, MCT4 immunoreactivity is observed in the SO, SR and SP. Scale bar=50 $\mu \mathrm{m}$. MCT4, monocarboxylate transporter 4; h, hour; d, days; SO, stratum oriens; SP, stratum pyramidale; $\mathrm{SR}$, stratum radiatum.

vibratome (Leica Microsystems $\mathrm{GmbH}$ ) and the hippocampus was cut out using a surgical blade. The hippocampal tissues were homogenized in $50 \mathrm{mM}$ PBS ( $\mathrm{pH}$ 7.4) containing $0.1 \mathrm{mM}$ EGTA (pH 8.0), 0.2\% Nonidet P-40, 10 mM EDTA (pH 8.0), $15 \mathrm{mM}$ sodium pyrophosphate, $100 \mathrm{mM} \beta$-glycerophosphate, $50 \mathrm{mM}$ sodium fluoride, $150 \mathrm{mM}$ sodium chloride, $2 \mathrm{mM}$ sodium orthovanadate, $1 \mathrm{mM}$ phenylmethylsulfonyl fluoride and $1 \mathrm{mM}$ dithiothreitol (DTT). Following centrifugation for $5 \mathrm{~min}$ at $16,000 \mathrm{x} \mathrm{g}$ at $4^{\circ} \mathrm{C}$, the protein concentration was determined in the supernatants using a Micro BCA protein assay kit with bovine serum albumin as the standard (Pierce; Thermo Fisher Scientific, Inc., Waltham, MA, USA). Aliquots containing $20 \mu \mathrm{g}$ of total protein were boiled in loading buffer containing $150 \mathrm{mM}$ Tris (pH 6.8), $3 \mathrm{mM}$ DTT, 6\% SDS, $0.3 \%$ bromophenol blue and $30 \%$ glycerol. Each aliquot was subsequently loaded onto a $12 \%$ polyacrylamide gel. Following electrophoresis, the gels were transferred to nitrocellulose membranes (Pall Life Sciences, Port Washington, NY, USA). To reduce background staining, the membranes were incubated with 5\% non-fat dry milk in PBS containing $0.1 \%$ Tween-20 for $45 \mathrm{~min}$ at $25^{\circ} \mathrm{C}$, which was followed by incubation with peroxidase-conjugated rabbit anti-MCT4 (1:500; catalog no. sc-50329, Santa Cruz Biotechnology, Inc.) and rabbit anti- $\beta$ actin (1:2,000; catalog no. ab8227; Abcam, Cambridge, UK) overnight at $4^{\circ} \mathrm{C}$ and an ECL chemiluminescent kit (Pierce; Thermo Fisher Scientific, Inc.). The blot was densitometrically scanned for the quantification of ROD of each band using Scion Image software version 4.0.3 (Scion Corporation., Frederick, MD). Expression was normalized against $\beta$-actin.

Statistical analysis. Data are presented as the mean + standard error of the mean. The differences among means were analyzed by one-way analysis of variance followed by Bonferroni's post-hoc correction to compare the time-dependent changes 


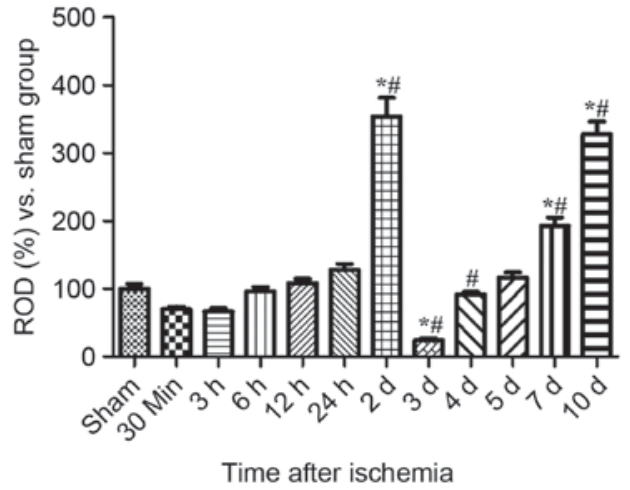

Figure 2. ROD of MCT4 immunoreactivity in the sham-operated group and at various time points following ischemia in ischemia-operated groups. RODs are expressed as a percentage of the value of MCT4 immunoreactivity in the sham-operated group of hippocampal CA1 region per section of the sham-operated and ischemia-operated groups. $\mathrm{n}=5$ per group, ${ }^{*} \mathrm{P}<0.05$ vs. sham-operated group and ${ }^{\#} \mathrm{P}<0.05$ vs. pre-adjacent time point group. Data are presented as the mean + standard error of the mean. ROD, relative optical density; MCT4, monocarboxylate transporter 4.

in MCT4 immunoreactivity. Analysis was performed using GraphPad Prism 5.01 software (GraphPad Software, Inc., La Jolla, CA, USA). $\mathrm{P}<0.05$ was considered to indicate a statistically significant difference.

\section{Results}

Changes of MCT4 immunoreactivity in the hippocampal CA1 region. Microscopy images are presented in Fig. 1 and quantification of immunoreactivity are presented in Fig. 2. MCT4 immunoreactivity was observed in the stratum pyramidale of the CA1 region of the sham-operated group (Fig. 1A). At $30 \mathrm{~min}$ and $3 \mathrm{~h}$ after ischemia, reduced MCT4 immunoreactivity was observed in the stratum pyramidale compared with the immunoreactivity observed in the sham-operated group (Figs. $1 \mathrm{~B}$ and $\mathrm{C}$ and 2). Between 6 and $24 \mathrm{~h}$ after ischemia, MCT4 immunoreactivity levels increased time-dependently in the CA1 region, however, the difference in the MCT4 immunoreactivity was not statistically different between sham and these groups (Figs. 1D-F and 2). MCT4 immunoreactivity was significantly increased 2 days following ischemia in the stratum pyramidale compared with the sham-operated group (Figs. 1G and 2). At 3 days following the treatment, MCT4 immunoreactivity decreased significantly in the stratum pyramidale compared with at 2 days following ischemia and was barely detectable in this region (Figs. $1 \mathrm{H}$ and 2). At days 4 and 5 following ischemia, MCT4 immunoreactivity increased in the strata oriens and radiatum compared with sham-operated animals (Fig. 1I and $\mathrm{J}$ ). However, few MCT4 immunoreactive structures were detected in the stratum pyramidale (Fig. 1I and J). At 7 and 10 days following ischemia, MCT4 immunoreactivity was observed in the strata oriens, radiatum and pyramidale (Fig. 1K and L). In these groups, MCT4 immunoreactivity levels significantly increased compared with the group at 5 days following ischemia $(\mathrm{P}<0.05$; Fig. 2). Numerous MCT4 immunoreactive structures were colocalized with activated (exhibiting a hypertrophied cytoplasm) GFAP immunoreactive astrocytes in the CA1 region of the hippocampus (Fig. 3).

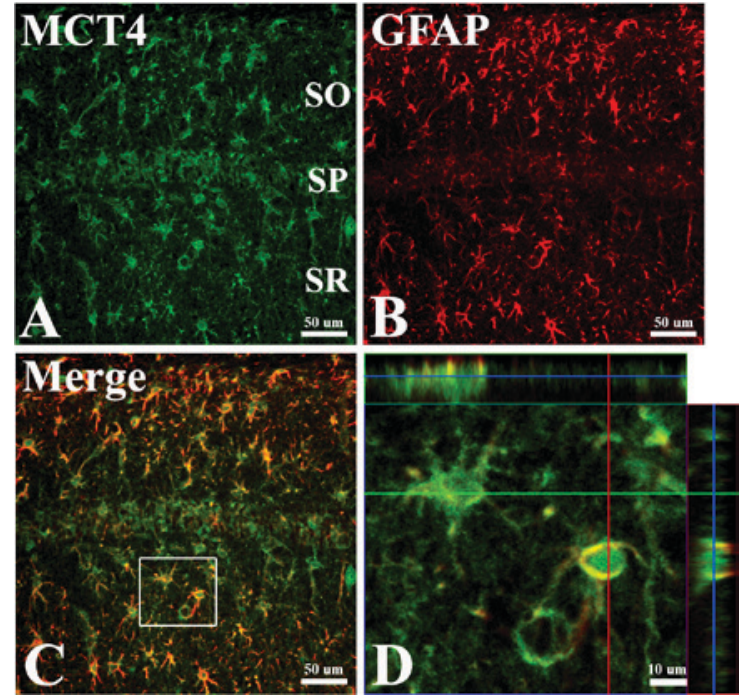

Figure 3. Double immunofluorescence staining for (A) MCT4, (B) GFAP, (C) merged images and (D) magnified image with orthogonal configuration in the hippocampal CA1 region 7 days following ischemia. Much of the MCT4 immunoreactivity is also detected in the GFAP immunoreactive astrocytes in the CA1 region. Green line, $\mathrm{x}$ axis; red line, y axis; blue line, position of the central panel image in the $\mathrm{z}$ stack. (A-C) Scale bar $=50 \mu \mathrm{m}$ and (D) scale bar=10 $\mu \mathrm{m}$. MCT4, monocarboxylate transporter; GFAP, glial fibrillary acidic protein SO, stratum oriens; SP, stratum pyramidale; SR, stratum radiatum.
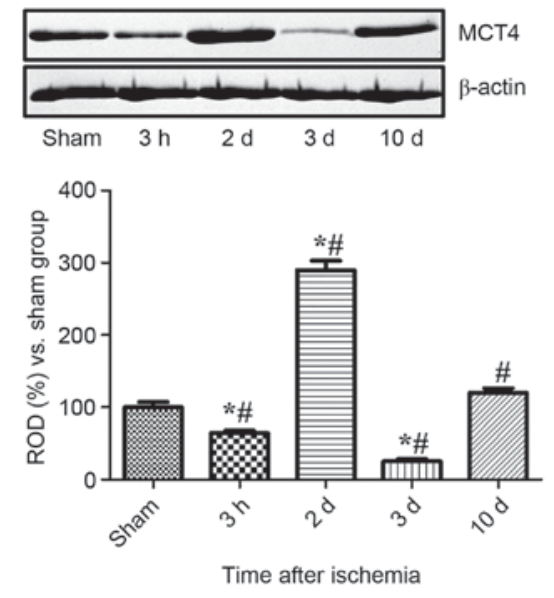

Figure 4. Western blot analysis. Protein levels are expressed as a percentage of MCT4 protein level normalized to $\beta$-actin protein level in the hippocampal homogenates of the sham- and ischemia-operated groups. $n=5$ per group, ${ }^{*} \mathrm{P}<0.05$ vs. sham-operated group and ${ }^{\#} \mathrm{P}<0.05$ vs. pre-adjacent time point group. Data are presented as the mean + standard error of the mean. MCT4, monocarboxylate transporter; d, days; ROD, relative optical density.

Changes of MCT4 protein levels in the hippocampal CAI region. MCT4 protein levels decreased significantly in hippocampal CA1 homogenates $3 \mathrm{~h}$ following ischemia compared with CA1 homogenates sampled from the sham-operated group $(\mathrm{P}<0.05)$. The protein levels of MCT4 exhibited a significant 2.9-fold increase 2 days following ischemia compared with the sham-operated group, and a 4.5 -fold increase at 2 days compared with the group at $3 \mathrm{~h}$ following ischemia. Only weak MCT4 bands were detected 3 days after ischemia and MCT4 levels were $25 \%$ of the sham-operated group levels. At 10 days after ischemia, MCT4 levels were marginally higher 
in the experimental group compared the sham-operated group (Fig. 4).

\section{Discussion}

Hypoxia-induced glycolysis promotes the synthesis of lactate in the brain. Under this pathological condition, lactate concentration in the extracellular space may undergo a 10-fold increase relative to a control brain (20-22). It has previously been suggested that lactate itself may be considered an important energy substrate, and that it may have an essential role in cellular metabolism (3) and memory formation $(23,24)$. Uptake of lactate by MCTs is operated by several isoforms of MCTs. In particular, MCT4 is expressed in the hippocampal CA1 pyramidal neurons of the Mongolian gerbil (13). The present study investigated the spatial and temporal changes of MCT4 expression in the hippocampal CA1 region. In the sham-operated group, MCT4 immunoreactivity was observed in the pyramidal cells of the hippocampal CA1 region. This result is consistent with findings previously reported by colleagues of the authors (13). In addition, MCT4 has been demonstrated as expressed in the neurons of $o b / o b$ obese mice, high-fat diet fed mice and also $d b / d b$ diabetic mice (11). However, MCT4 immunoreactivity is observed in the astrocytes of control rats and mice (9-12). This observation may be associated with characteristic factors in plasma lipid response to dietary carbohydrate and fatty acids previously reported in Mongolian gerbils; Mongolian gerbils have been reported to be more sensitive to fatty acids in the presence and absence of dietary cholesterol (25-27).

The present study observed the fluctuation of MCT4 levels and immunoreactivity following the induction of transient forebrain ischemia. MCT4 levels were significantly increased 2 days after ischemia in the CA1 pyramidal cells and significantly decreased in this region 3 days after ischemia, compared with the sham-operated group. The sharp decrease in MCT4 levels observed in the hippocampal CA1 region may be associated with the ischemia-induced neuronal damage that has been previously detected in the hippocampal CA1 region. Inhibition of lactate transport induced by blocking of MCT has been reported to exacerbate neuronal damage in different rat models of cerebral ischemia $(28,29)$, while ischemic preconditioning significantly increased MCT4 immunoreactivity in the pyramidal cells of the CA1 region (13). Following the ischemia-induced decrease in MCT4 levels, MCT4 immunoreactivity was increased in the astrocytes of the CA1 region. The initial decrease in MCT4 expression may be associated with the increased use of MCT4 aimed at reducing the ischemia-induced increase in lactate levels. The subsequent rise in MCT4 levels may be interpreted as a compensatory response to the ischemic damage. The elevated levels of MCT4 may be due to an increase in the synthesis of MCT4 in the pyramidal cells and an upregulation of MCT4 activity in astrocytes caused by ischemia-induced neuronal death. The results are supported by previous studies that demonstrated that MCT4 immunoreactivity is increased $1 \mathrm{~h}$ post-ischemia and is no longer detected $24 \mathrm{~h}$ after the treatment, which suggests neuronal death in the brain by middle cerebral artery occlusion (14). At 5 days following ischemia, MCT4 immunoreactivity is significantly increased within and around the infarct zone of permanent focal ischemia in rats (16).

In conclusion, the results of the present study indicated that MCT4 is expressed in the pyramidal cells of the hippocampal CA1 region of the Mongolian gerbil, and the location and immunoreactivity of MCT4 undergo time-dependent changes that aim to remove or process the lactate in the CA1 region. Acute reduction in MCT4 may be associated with neuronal damage in the hippocampal CA1 region. The present study demonstrated that time-dependent expressional changes in MCT4 after transient ischemia may be involved in lactate metabolism after ischemic damage in the hippocampus.

\section{Acknowledgements}

The authors would like to thank to Dr Seung-Hae Kwon of the Korean Basic Science Institute Chuncheon Center (Daejeon, Korea) for technical assistance with the confocal image analyses. This work was supported by the Basic Science Research Program through the National Research Foundation of Korea, funded by the Ministry of Education (grant no. NRF-2013R1A1A2059364). In addition, the present study was supported by the Research Institute for Veterinary Science of Seoul National University.

\section{References}

1. Boumezbeur F, Petersen KF, Cline GW, Mason GF, Behar KL, Shulman GI and Rothman DL: The contribution of blood lactate to brain energy metabolism in humans measured by dynamic 13C nuclear magnetic resonance spectroscopy. J Neurosci 30: 13983-13991, 2010.

2. Wyss MT, Jolivet R, Buck A, Magistretti PJ and Weber B: In vivo evidence for lactate as a neuronal energy source. J Neurosci 31: 7477-7485, 2011.

3. Schurr A: Lactate: The ultimate cerebral oxidative energy substrate? J Cereb Blood Flow Metab 26: 142-152, 2006.

4. Brooks GA: Lactate: Link between glycolytic and oxidative metabolism. Sports Med 37: 341-343, 2007.

5. Halestrap AP and Price NT: The proton-linked monocarboxylate transporter (MCT) family: Structure, function and regulation. Biochem J 343 Pt 2: 281-299, 1999.

6. Pierre K and Pellerin L: Monocarboxylate transporters in the central nervous system: Distribution, regulation and function. J Neurochem 94: 1-14, 2005.

7. Gerhart DZ, Enerson BE, Zhdankina OY, Leino RL and Drewes LR: Expression of monocarboxylate transporter MCT1 by brain endothelium and glia in adult and suckling rats. Am J Physiol 273: E207-E213, 1997.

8. Hanu R, McKenna M, O'Neill A, Resneck WG and Bloch RJ: Monocarboxylic acid transporters, MCT1 and MCT2, in cortical astrocytes in vitro and in vivo. Am J Physiol Cell Physiol 278: C921-C930, 2000.

9. Pierre K, Pellerin L, Debernardi R, Riederer BM and Magistretti PJ: Cell-specific localization of monocarboxylate transporters, MCT1 and MCT2, in the adult mouse brain revealed by double immunohistochemical labeling and confocal microscopy. Neuroscience 100: 617-627, 2000.

10. Rafiki A, Boulland JL, Halestrap AP, Ottersen OP and Bergersen L: Highly differential expression of the monocarboxylate transporters MCT2 and MCT4 in the developing rat brain. Neuroscience 122: 677-688, 2003.

11. Pierre K, Parent A, Jayet PY, Halestrap AP, Scherrer U and Pellerin L: Enhanced expression of three monocarboxylate transporter isoforms in the brain of obese mice. J Physiol 583: 469-486, 2007

12. Gao C, Wang C, Liu B, Wu H, Yang Q, Jin J, Li H, Dong S, Gao G and Zhang $\mathrm{H}$ : Intermittent hypoxia preconditioning-induced epileptic tolerance by upregulation of monocarboxylate transporter 4 expression in rat hippocampal astrocytes. Neurochem Res 39: 2160-2169, 2014. 
13. Hong S, Ahn JY, Cho GS, Kim IH, Cho JH, Ahn JH, Park JH, Won MH, Chen BH, Shin BN, et al: Monocarboxylate transporter 4 plays a significant role in the neuroprotective mechanism of ischemic preconditioning in transient cerebral ischemia. Neural Regen Res 10: 1604-1611, 2015.

14. Rosafio K, Castillo X, Hirt L and Pellerin L: Cell-specific modulation of monocarboxylate transporter expression contributes to the metabolic reprograming taking place following cerebral ischemia. Neuroscience 317: 108-120, 2016

15. Tseng MT, Chan SA and Schurr A: Ischemia-induced changes in monocarboxylate transporter 1 reactive cells in rat hippocampus. Neurol Res 25: 83-86, 2003.

16. Zhang F, Vannucci SJ, Philp NJ and Simpson IA: Monocarboxylate transporter expression in the spontaneous hypertensive rat: Effect of stroke. J Neurosci Res 79: 139-145, 2005.

17. Moreira TJ, Pierre K, Maekawa F, Repond C, Cebere A Liljequist $\mathrm{S}$ and Pellerin L: Enhanced cerebral expression of MCT1 and MCT2 in a rat ischemia model occurs in activated microglial cells. J Cereb Blood Flow Metab 29: 1273-1283, 2009

18. National Research Council: Guide for the Care and Use of Laboratory Animals, 7th edition. National Academy Press, Washington DC, 1996.

19. Loskota WA, Lomax P and Verity MA: A stereotaxic atlas of the Mongolian Gerbil Brain (Meriones unguiculatus). Ann Arbor Science Publishers Inc., Ann Arbor, pp70-79, 1974.

20. Rehncrona S, Rosén I and Siesjö BK: Brain lactic acidosis and ischemic cell damage: 1 . Biochemistry and neurophysiology. J Cereb Blood Flow Metab 1: 297-311, 1981.

21. Folbergrová J, Memezawa H, Smith ML and Siesjö BK: Focal and perifocal changes in tissue energy state during middle cerebral artery occlusion in normo- and hyperglycemic rats. J Cereb Blood Flow Metab 12: 25-33, 1992.
22. Wagner KR, Kleinholz M, de Courten-Myers GM and Myers RE: Hyperglycemic versus normoglycemic stroke: Topography of brain metabolites, intracellular $\mathrm{pH}$, and infarct size. J Cereb Blood Flow Metab 12: 213-222, 1992.

23. Bouzier-Sore AK, Voisin P, Canioni P, Magistretti PJ and Pellerin L: Lactate is a preferential oxidative energy substrate over glucose for neurons in culture. J Cereb Blood Flow Metab 23: 1298-1306, 2003

24. Newman LA, Korol DL and Gold PE: Lactate produced by glycogenolysis in astrocytes regulates memory processing. PLoS One 6: e28427,2011.

25. Mercer NJ and Holub BJ: Response of free and esterified plasma cholesterol levels in the Mongolian gerbil to the fatty acid composition of dietary lipid. Lipids 14: 1009-1014, 1979.

26. Andersen DB and Holub BJ: Effects of dietary cholesterol level and type of dietary carbohydrate on hepatic and plasma sterols in the gerbil. Can J Physiol Pharmacol 60: 885-892, 1982.

27. Dictenberg JB, Pronczuk A and Hayes KC: Hyperlipidemic effects of trans fatty acids are accentuated by dietary cholesterol in gerbils. J Nutr Biochem 6: 353-361, 1995.

28. Schurr A, Payne RS, Miller JJ, Tseng MT and Rigor BM: Blockade of lactate transport exacerbates delayed neuronal damage in a rat model of cerebral ischemia. Brain Res 895: 268-272, 2001.

29. Wang Y, Guo SZ, Bonen A, Li RC, Kheirandish-Gozal L, Zhang SX, Brittian KR and Gozal D: Monocarboxylate transporter 2 and stroke severity in a rodent model of sleep apnea. J Neurosci 31: 10241-10248, 2011. 